\title{
Varia ou la preuve de la variété des questions environnementales
}

\section{Frédéric Chavy}

\section{(2) OpenEdition}

1 Journals

\section{Édition électronique}

URL : http://journals.openedition.org/developpementdurable/9141

DOI : 10.4000/developpementdurable.9141

ISSN : $1772-9971$

Éditeur

Association DD\&T

\section{Référence électronique}

Frédéric Chavy, «Varia ou la preuve de la variété des questions environnementales », Développement durable et territoires [En ligne], Vol. 2, n 3 | Décembre 2011, mis en ligne le 05 décembre 2011, consulté le 22 septembre 2020. URL : http://journals.openedition.org/developpementdurable/9141 ; DOI: https://doi.org/10.4000/developpementdurable.9141

Ce document a été généré automatiquement le 22 septembre 2020.

Développement Durable et Territoires est mis à disposition selon les termes de la licence Creative Commons Attribution - Pas d'Utilisation Commerciale 4.0 International. 


\title{
Varia ou la preuve de la variété des questions environnementales
}

\author{
Frédéric Chavy
}

1 Ce numéro de Développement Durable et Territoires est, et cela devrait devenir récurrent, majoritairement composé d'articles issus de la rubrique "Varia». Cela ne signifie nullement que notre intérêt pour les dossiers thématiques s'amenuise. D'ailleurs, plusieurs appels à contributions ont récemment été lancés sur les thèmes de la biodiversité et de l'aménagement du territoire, des relations santé-environnement, et des approches en écologie industrielle et économie de la fonctionnalité. Cette publication «Varia » est tout simplement l'indice que nous recevons de plus en plus de propositions spontanées et que la qualité générale de ces propositions tend à augmenter. Ainsi, dans le présent numéro, seront abordés des sujets variés comme : la participation du public à la nature (Jean-Eudes Beuret), l'évaluation des services rendus par les écosystèmes en France (Bruno Maresca, Xavier Mordret, Anne Lise Ughetto et Philippe Blancher), la forme transmoderne des pratiques récréatives de nature (Jean Corneloup), les sports de nature en débat (Yohann Rech et Jean-Pierre Mounet), les prospectives politiques de la gestion de l'eau en Europe (Sara Fernandez, Gabrielle Bouleau et Sébastien Treyer) ou encore la politisation du discours sur la gestion intégrée des zones côtières (Céline Ségalini). Chacun pourra y trouver matière à satisfaire sa curiosité intellectuelle et à étancher sa soif de connaissances.

2 Ce numéro est, par ailleurs, étoffé par un mini-dossier intitulé : «Développement durable: question de sens", coordonné par Elisabeth Michel-Guillou et Adeline Raymond dans lequel vous pourrez lire trois articles: le premier porte sur le développement durable en Amérique du Sud - en général - et l'expérience colombienne - en particulier (Oscar Navarro) ; le deuxième s'interroge sur le sens donné au concept de «durabilité " par la presse régionale au travers du discours des agriculteurs (Lise Hihannic et Elisabeth Michel-Guillou ); le dernier traite de la prise en compte de la durabilité dans la représentation sociale de la croissance économique (Hélène Blanc et Adeline Raymond). 
3 Sans oublier, notre rubrique habituelle « Lectures ». Même s'il est vrai que la rubrique « Point de vue » n'est pas représentée dans cette « fournée », elle n'est pas, pour autant, laissée à l'abandon. Rappelons que cette rubrique est destinée à accueillir des prises de positions, qui s'appuie sur une démonstration rigoureuse, mais dont la tonalité et l'intention peuvent déroger à l'exigence stricte d'un article scientifique. Cyril Decouzon, nouvellement accueilli au sein du Comité de rédaction, est dorénavant est chargé de cette rubrique.

4 Pour être complet, signalons la tenue récente, le 25 novembre 2011, de la cinquième Journée Interdisciplinaire sur le Développement Durable, sur le thème: " Reterritorialisation et développement durable : contraintes écologiques et logiques sociales » au cours de laquelle six interventions se sont tenues autour des enjeux posés par les questions de la reterritorialisation et notamment les circuits courts alimentaires. Cette journée donnera lieu à une valorisation dans notre revue.

5 La sixième Journée Interdisciplinaire sur le Développement Durable est déjà programmée pour le printemps 2012. À l'occasion du vingtième anniversaire du Sommet de Rio, la JIDD aura pour thème: "Le développement durable: concept sousexploité ou concept dépassé?"

6 Merci de nous suivre régulièrement et bonne lecture ! 